\title{
EL GÉNERO THAUMATOMYRMEX MAYR 1887 EN MÉXICO (HYMENOPTERA: FORMICIDAE)
}

\author{
Patricia RoJAS-FERNÁNDEZ \\ Departamento Biología de Suelos. Instituto de Ecología A. C., Xalapa, Ver. MEXICO. \\ patricia.rojas@inecol.edu.mx
}

Rojas-Fernández, P. 2009. El género Thaumatomyrmex Mayr 1887 en México (Hymenoptera: Formicidae). Acta Zool. Mex (n. s.) 25(1): 61-70.

RESUMEN. En este trabajo se analiza la presencia del género Thaumatomyrmex en México. La especie encontrada en Los Tuxtlas, Veracruz, es el registro más norteño del género en América. Debido a la falta de definición en los caracteres diagnósticos de las especies del grupo ferox, no fue posible asignar con certeza un nombre específico al material, pudiendo ser tanto T. ferox como T. atrox. Se incluyen fotografías y medidas corporales de utilidad taxonómica de los dos ejemplares colectados. Finalmente se discute la rareza del género en México.

Palabras clave: hormigas, Formicidae, Veracruz, México, rareza.

Rojas-Fernández, P. 2009. The genus Thaumatomyrmex Mayr 1887 in Mexico (Hymenoptera: Formicidae). Acta Zool. Mex (n. s.) 25(1): 61-70.

ABSTRACT. In this paper the distribution in Mexico of the ant genus Thaumatomyrmex is analyzed. The Los Tuxtlas region in the state of Veracruz represents the most northern locality for this rare Neotropical genus. Body measurements and photographs of the two specimens collected are provided; the status of the species remains unclear due to the lack of definition on the species diagnostic characters. Rarity of the genus in Mexico is discussed.

Key words: ants, Formicidae, Veracruz, Mexico, rarity.

\section{INTRODUCCIÓN}

Las hormigas del género Thaumatomyrmex (Ponerinae: Thaumatomyrmecini) tienen una morfología extraordinaria caracterizada por su cabeza trapezoidal con la parte anterior más ancha que la posterior y sus grandes mandíbulas delgadas, curvas y con tres dientes largos y agudos.

Desde la descripción del género y la especie T. mutilatus por Mayr en 1887 se han descrito 11 especies más, tres de las cuales (paludis, manni y zeteki) fueron sinonimizadas por Longino (1988). Kempf (1975) dividió el género en tres grupos de especies, cada uno con una distribución geográfica particular: 1) grupo mutilatus con dos especies endémicas del sureste de Brasil; 2) grupo cochlearis con cuatro especies endémicas de Cuba y 3) grupo ferox con dos especies (T. atrox Weber y T. ferox Mann) de amplia distribución en el Neotrópico.

Estas hormigas tienen colonias muy pequeñas (3-12 individuos), anidan en la hojarasca y entre las raíces de plantas en diversos tipos de vegetación desde selvas 
húmedas, hasta bosques secos (Jahyni et al. 2007). Son depredadoras muy especializadas y capturan exclusivamente milpiés polixénidos a los que despojan de sus sedas urticantes antes de darlos como alimento a las larvas (Brandão et al. 1991). Aunque este comportamiento ha sido bien estudiado solamente en T. contumax Kempf, es probable que las otras especies tengan hábitos similares.

Las especies de Thaumatomyrmex se recolectan muy raramente; por lo general se obtienen individuos aislados a partir de hojarasca procesada con embudos de Berlese o extractores de Winkler. La excepción a este patrón son las colectas de decenas de individuos en una localidad en el sureste de Brasil (Delabie et al. 2000). No obstante esto, el número total de ejemplares en las colecciones del mundo apenas sobrepasa los 200 y de algunas especies solamente se conocen los especímenes tipo (bariay Fontenla, nageli B.Urbani \& De Andrade). La especie más común es atrox con registros de siete países desde Costa Rica hasta Brasil.

En este trabajo se registra por primera vez la presencia del género Thaumatomyrmex en el estado de Veracruz, México. Se analiza la posible identidad específica de los dos ejemplares colectados, utilizando las medidas corporales y características de la cutícula propuestas por Kempf (1975). También se discute la distribución geográfica del género y su rareza en México.

\section{MATERIAL Y MÉTODOS}

El registro de Thaumatomyrmex se hace con base en dos obreras colectadas en la Reserva de la Biosfera de Los Tuxtlas, Veracruz, localizada en la parte central de la costa del Golfo de México.

Ejemplar No. 1. MEXICO: Veracruz, Estación de Biología Tropical Los Tuxtlas, Mpio. Catemaco, 18 35'99'N 9504'99'W, 150 m snm, 19-IX-1993, J. Bueno. BSIE-5169. Muestra de hojarasca de $0.25 \mathrm{~m}^{2}$, flotación. El tipo de vegetación de este sitio es selva alta perennifolia con $4500 \mathrm{~mm}$ de precipitación y una temperatura media anual de $24^{\circ} \mathrm{C}$. La muestra en la que se encontró el ejemplar pertenece a un conjunto de 50 cuadrantes de hojarasca tomados en el sitio y procesados con la técnica de flotación (Edwards 1991).

Ejemplar No. 2. MEXICO: Veracruz, Ejido San Fernando, Mpio. Soteapan, $18^{\circ} 16^{\prime} 87^{\prime} \mathrm{N} 94^{\circ} 53$ ' 32'”, $1029 \mathrm{~m}$ snm, 5-XII-2003, A. Angeles y P. Rojas. BSIE5170. Muestra de hojarasca de $1 \mathrm{~m}^{2}$, Winkler. El sitio es un cafetal abandonado ubicado en un tipo de vegetación transicional entre selva alta perennifolia y bosque mesófilo de montaña, con $1200 \mathrm{~mm}$ de precipitación y una temperatura media anual de $23^{\circ} \mathrm{C}$. La muestra en la que se encontró el ejemplar pertenece a un conjunto de 300 cuadrantes de hojarasca tomados en diferentes tipos de vegetación dentro de la Reserva y procesados con sacos mini-Winkler (Fisher 1998).

La distancia aproximada entre los dos sitios de colecta es de $40 \mathrm{~km}$. Los ejemplares se montaron en seco y están depositados en la colección de Formicidae 
del Departamento Biología de Suelos del Instituto de Ecología, A.C. (BSIE). Todas las medidas fueron tomadas a $50 \mathrm{X}$ con un estereomicroscopio Zeiss con micrómetro ocular.

Las medidas e índices se obtuvieron siguiendo a Kempf (1975):

Longitud de la cabeza (HL): en vista frontal, la distancia entre dos líneas paralelas trazadas a nivel del extremo anterior de los lóbulos frontales y a nivel de la parte posterior de los lóbulos occipitales.

Ancho de la cabeza (HW): en vista frontal, ancho máximo de la cabeza.

Longitud de la mandíbula (ML): longitud de la cuerda de la mandíbula, excluyendo el cóndilo articular basal.

Longitud del escapo (SL): longitud de la cuerda del escapo antenal, excluyendo el cóndilo articular y su conexión con el escapo en sí.

Longitud de Weber (WL): de perfil, longitud del mesosoma desde la parte anterior del pronoto, excluyendo el cuello, hasta el ángulo metasternal.

Ancho del peciolo (PW): en vista dorsal, ancho máximo del peciolo.

Longitud total (TL): HL+WL+longitud del peciolo+ longitud del gáster, excluyendo el aguijón.

Indice cefálico (CI): HW/HL x 100

Indice mandibular (MI): ML/HL x 100

Indice del escapo (SI): SL/HL x 100

\section{RESULTADOS}

De manera preliminar y siguiendo la clave de Kempf (1975) he identificado a las dos obreras colectadas como T. ferox. Sin embargo, los criterios de separación de las especies del subgrupo 2 del grupo ferox, al que pertenece nuestra especie, aún no están claros. Los dos ejemplares son muy similares en cuanto a pilosidad, color, forma de las mandíbulas y en que ambos carecen de un dientecillo basal en las mandíbulas (Figs. 1a y 2a). Las diferencias entre ambos son el tamaño del cuerpo (Cuadro 1) y la microescultura de la cutícula. El ejemplar de la Estación (1) es más pequeño y presenta en todo el cuerpo "...fine, short vermiculate impressions resembling a very sparse, appresed pubescence" (Weber 1939, p. 98), mientras que el ejemplar de San Fernando (2) es de mayor tamaño y presenta estas marcas únicamente en la parte inferior de la cara anterior del peciolo y en la cara anterior del terguito I del gáster, en donde con adecuada iluminación pueden verse. De manera interesante, los dos ejemplares difieren en todas las medidas tomadas, excepto en el índice cefálico (CI), que es idéntico (Cuadro 1). 
Rojas-Fernández, Thaumatomyrmex en México
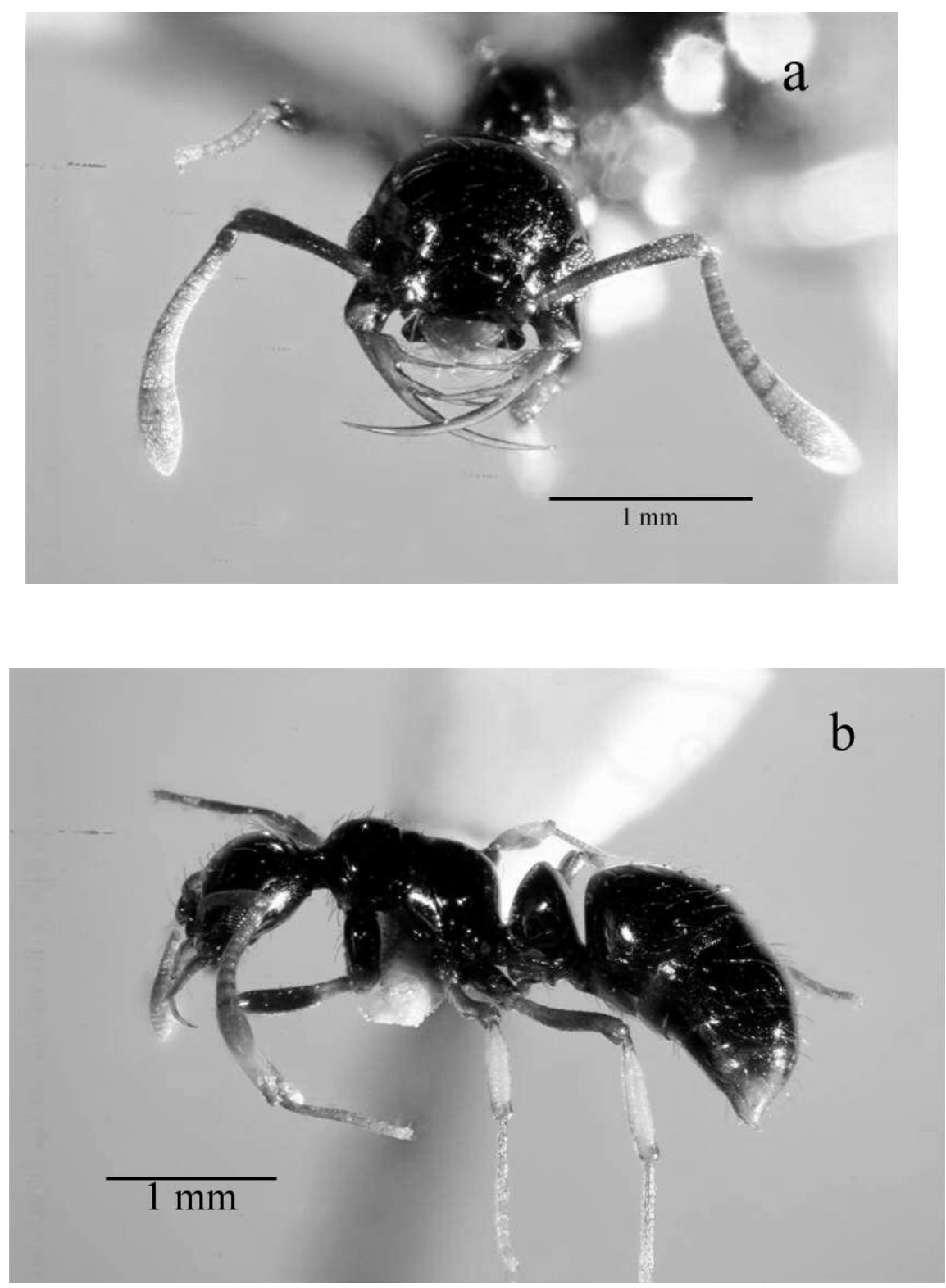

Figura 1. Thaumatomyrmex ferox Mann (¿atrox Weber?) de Los Tuxtlas, Veracruz, México. Ejemplar de la Estación. Cabeza en vista frontal (a) y cuerpo de perfil (b). 

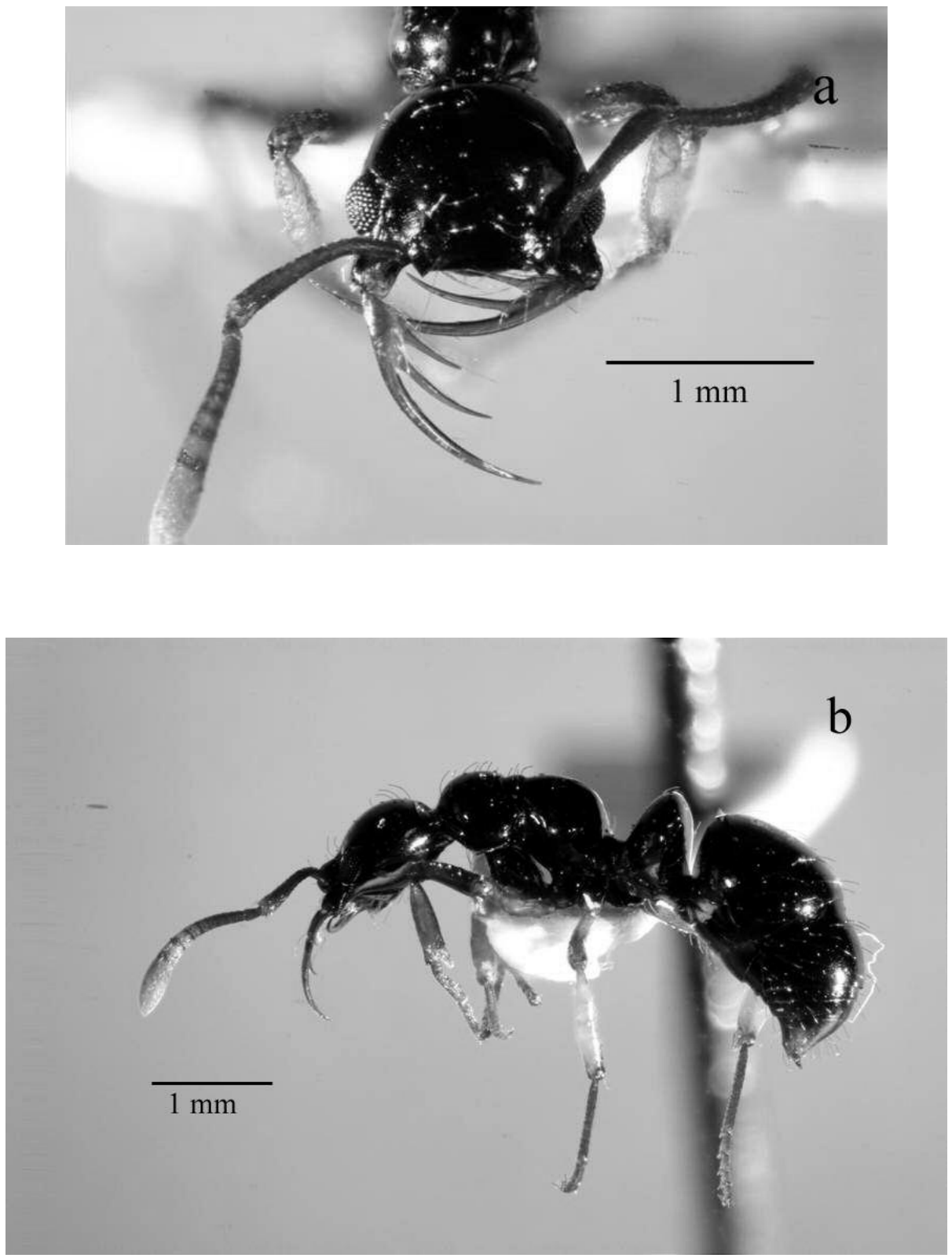

Figura 2. Thaumatomyrmex ferox Mann (¿atrox Weber?) de Los Tuxtlas, Veracruz, México. Ejemplar de San Fernando. Cabeza en vista frontal (a) y cuerpo de perfil (b). 
Rojas-Fernández, Thaumatomyrmex en México

Cuadro 1. Medidas e índices de T. atrox, T. ferox (tomadas de Kempf 1975) y de los ejemplares estudiados en este trabajo (Estación y S. Fernando). TL= largo total, $\mathrm{HL}=$ largo de la cabeza, $\mathrm{HW}=$ ancho de la cabeza, $\mathrm{CI}=$ índice cefálico $(\mathrm{HW} / \mathrm{HLx} 100), \mathrm{ML}=$ largo de la mandíbula, $\mathrm{MI}=$ índice mandibular (ML/HLx100), SL= largo del escapo, SI= índice del escapo (SL/HLx100), WL= longitud de Weber, $\mathrm{PW}=$ ancho del pecíolo.

\begin{tabular}{ccccc}
\hline & ferox & atrox & Estación & S. Fernando \\
\hline TL & 4.7 & 4.4 & 4.1 & 4.4 \\
HL & 0.89 & 0.91 & 0.87 & 0.97 \\
HW & 1.13 & 1.09 & 1.05 & 1.17 \\
CI & 127 & $\mathbf{1 2 0}$ & $\mathbf{1 2 0}$ & $\mathbf{1 2 0}$ \\
ML & 1.20 & 1.15 & 1.01 & 1.30 \\
MI & 134 & 126 & 116 & 134 \\
SL & 0.77 & 0.80 & 0.78 & 0.89 \\
SI & 87 & 88 & 89 & 91 \\
WL & 1.36 & 1.33 & 1.17 & 1.32 \\
PW & 0.73 & 0.73 & 0.68 & 0.78 \\
\hline
\end{tabular}

Con el registro del género en el estado de Veracruz, se extiende su límite norte de distribución en tierras continentales de América. El registro publicado más norteño anterior a éste es el de Bestelmeyer et al. (2000) quienes encontraron a T. ferox en el Parque Nacional Laguna del Tigre en Petén, Guatemala.

\section{DISCUSIÓN}

La especie de Los Tuxtlas: iferox o atrox?. El grupo de especies ferox se caracteriza por tener la superficie del cuerpo muy lisa y brillante, con algunas arrugas o surcos restringidos a los lóbulos frontales, el cuello y la base de las mandíbulas. El grupo comprende a la fecha dos especies válidas: ferox y atrox. Kempf (1975) separa las dos especies únicamente por la presencia (atrox) o no (ferox) de un pequeño diente basal en las mandíbulas y dice que "atrox is dangerously close to ferox". También señala que la proporción entre largo y ancho de la cabeza (índice cefálico) podría ser un carácter importante a nivel específico. Más tarde Longino (1988) revisa el grupo, pero mantiene la situación de las dos especies igual, separándolas únicamente por la presencia o no del diente basal. Sin embargo ahora se sabe, con base en más ejemplares encontrados principalmente en Panamá y Costa Rica, que ferox puede también presentar el dientecillo basal en la mandíbula (Longino 2005). Esto confirma que este carácter es variable y de poco valor taxonómico (Baroni Urbani y De Andrade 2003). 
Los dos ejemplares de la especie de Los Tuxtlas son muy similares entre sí y carecen del dientecillo basal mandibular (Fig. 1 y 2), por lo que deberían ser reconocidos como ferox. Por otro lado, junto con la microescultura de la cutícula, la principal diferencia entre los dos ejemplares está en el tamaño del cuerpo. Los ejemplares difieren en todas las medidas tomadas excepto en el índice cefálico (CI), que es idéntico. Esto apoya la propuesta de Kempf respecto a que la forma de la cabeza puede ser un carácter útil para separar a las especies, pero no la de Longino acerca del tamaño del cuerpo (Cuadro 1). Al comparar el CI de los ejemplares mexicanos con los que proporciona Kempf para atrox y ferox, se encuentra que su valor es igual al de atrox y no al de ferox. Si se toma este carácter como válido, entonces la especie de Los Tuxtlas es atrox.

Otros caracteres como las cóstulas concéntricas alrededor de los ojos, las microornamentaciones de la cutícula y el patrón de sedas deben estudiarse en ejemplares de las dos especies, ya que unos varían de forma continua y otros de manera discreta. Posiblemente atrox y ferox sean una misma especie con una gran variación intraespecífica. En el trabajo más reciente sobre el género, Jahyni et al. (2007) mantienen la posición de que es necesario recolectar un mayor número de ejemplares para poder discriminar con exactitud las especies de Thaumatomyrmex.

Límite norte del género. La distribución geográfica de ferox va desde México hasta la amazonia brasileña, mientras que la de atrox desde Panamá hasta el sureste de Brasil y Bolivia. La distribución de ambas especies se sobrelapa a la altura de Costa Rica y Panamá por lo que las colectas en esta región deberían ser prioritarias. La presencia de Thaumatomyrmex en México y particularmente en Los Tuxtlas es interesante desde el punto de vista biogeográfico, ya que lo ubica dentro del grupo de géneros de hormigas de origen sudamericano que alcanzan su límite norte de distribución en las tierras bajas y de mediana altitud del estado de Veracruz (Lattke 2003), siguiendo la distribución de las selvas húmedas en el continente. Es poco probable que este género se encuentre más al norte si se considera que solamente quedan pequeños parches muy perturbados de selva más allá del paralelo $18^{\circ} \mathrm{N}$, y que Los Tuxtlas ha sido establecido como el límite boreal actual de la selva húmeda tropical (Dirzo y Miranda 1991). Recientemente se incluyeron en la página de internet AntWeb (2008) registros de T. ferox provenientes del estado de Chiapas y de una especie no identificada del estado de Guerrero, ambas localidades ubicadas al sur de Los Tuxtlas.

Rareza. Un factor estrechamente ligado con la captura de estas hormigas es su aparente rareza en la mayoría de los sitios en los que se le ha encontrado. Aunque pueden ser localmente abundantes en ciertos sitios como los reportados por Delabie et al. (2000), no parece ser la generalidad. En nuestro caso, el ejemplar colectado en San Fernando proviene de un muestreo de hormigas del suelo realizado en 60 sitios dentro de la Reserva, incluyendo bosques no perturbados y diversos 
agroecosistemas. De varios miles de individuos obtenidos de 300 muestras de hojarasca ( $1 \mathrm{~m}^{2}$, Winkler), 180 trampas de caída y 60 monolitos de suelo $(25 \times 25 \times 30$ $\mathrm{cm}$ ) solamente se capturó un ejemplar de Thaumatomyrmex en una de la muestras de hojarasca. El ejemplar de la Estación fue capturado mediante la técnica de flotación, método no utilizado convencionalmente para capturar hormigas. Por otro lado, en esta misma región se han realizado con anterioridad muestreos intensivos sin que se haya encontrado ningún ejemplar de este género (Cartas 1992, Quiroz y Valenzuela 1995). Los recientes registros en internet de Chiapas y Guerrero están representados por un solo ejemplar, lo que apoya la rareza de estas hormigas en México.

Probablemente Delabie et al. (2000) tengan razón en afirmar que no se han utilizado los métodos adecuados para capturar a estas especies, sin embargo, para el caso de nuestras localidades tampoco los sacos Winkler parecen ser lo más idóneo. Para determinar si esta especie es localmente rara o no, deberemos hacer búsquedas minuciosas dirigidas a ciertos microhábitats como pequeñas cañadas en la selva, la base de los árboles, dentro o debajo de troncos podridos, etc. Así como el uso rutinario de los sacos Winkler ha mostrado que algunas especies que parecían ser raras en realidad son comunes, la utilización de un método ad hoc para encontrar los nidos de Thaumatomyrmex nos esclarecerá si son raras o simplemente no hemos buscado adecuadamente.

AGRADECIMIENTOS. A Antonio Angeles y Lizbeth Hernández por su apoyo en el trabajo de campo. Parte de este trabajo fue apoyado por el proyecto BGBD-MEXICO/TSBF/PNUMA/GEF.

\section{LITERATURA CITADA}

AntWeb. The California Academy of Sciences. 2008. http://www.antweb.org., (acceso: 15/X/08).

Baroni Urbani, C. \& M.L De Andrade. 2003. The ant genus Thaumatomyrmex in Cuba (Hymenoptera: Formicidae) with description of two new species. Bulletin de la Société Entomologique Suisse, 76:263-277.

Bestelmeyer, B., L.A. Alonso \& R.R. Snelling. 2000. The ants (Hymenoptera: Formicidae) of Laguna del Tigre National Park, Peten, Guatemala. Conservation International. RAP Bulletin \& Biological Assesment, 16:75-83.

Brandao, C.R.F., J.L.M. Diniz \& E.M. Tomotake. 1991. Thaumatomyrmex strips millipedes for prey, a novel predatory behaviour in ants and the first case of sympatry in the genus. Insectes Sociaux, 38:335-344.

Cartas, C.A. 1993. Aspectos Ecológicos de la Formicofauna (Hymenoptera: Formicidae) del Volcán San Martín Pajapan, Ver. Tesis de Licenciatura. Facultad de Biología. Universidad Veracruzana. 78 pp.

Delabie, J.H.C., D.F. Fresnau \& A. Pezon. 2000. Notes on the ecology of Thaumatomyrmex spp. (Hymenoptera: Formicidae: Ponerinae) in Southeast Bahia, Brazil. Sociobiology, 36:571-584.

Dirzo, R. \& A. Miranda. 1991. El límite boreal de la selva tropical húmeda en el continente americano. Contracción de la vegetación y solución de una controversia. Interciencia, 16:240-247.

Edwards, C.A. 1991. The assessment of populations of soil-inhabiting invertebrates. Agriculture, Ecosytems \& Environment, 34:145-176. 
Fisher, B.L. 1998. Ant diversity patterns along an elevational gradient in the Réserve Spéciale d'Anjanaharibe-Sud and on the western Masaola Peninsula, Madagascar. Fieldiana: Zoology (n.s.), 90:3967.

Jahyni, B., S. Lacau, J.H.C. Delabie \& D. Fresnau. 2007. Le genre Thaumatomyrmex Mayr 1887, cryptique et prédateur spécialiste de Diplopoda Penicillata. Pp. 329-346. In: E. Jiménez, F. Fernández, T.M. Arias \& F.H. Lozano-Zambrano (eds.). Sistemática, biogeografía y conservación de las hormigas cazadoras de Colombia. Instituto de Investigación de Recursos Biológicos Alexander von Humboldt. Bogotá.

Kempf, W.W. 1975. A revision of the Neotropical ponerine ant genus Thaumatomyrmex Mayr (Hymenoptera: Formicidae). Studia Entomologica, 18:95-126.

Lattke, J. 2003. Biogeografía de las hormigas neotropicales. Pp. 65-85. In: Fernández, F. (ed.). Introducción a las hormigas de las región Neotropical. Instituto de Investigación de Recursos Biológicos Alexander von Humboldt, Bogotá.

Longino, J.T. 1988. Notes on the taxonomy of the neotropical ant genus Thaumatomyrmex Mayr (Hymenoptera: Formicidae). Pp. 35-42. In: Trager, J.C. (ed.). Advances in Myrmecology. E.J. Brill, New York.

Longino, J.T. 2005. The genus Thaumatomyrmex in Costa Rica. Documento World Wide Web: http://academic.evergreen.edu/projects/ants/genera/thaumatomyrmex/specieslist.html, (acceso:15/VIII/08).

Mayr, G. 1887. Sudamerikanische Formiciden. Verhandlungen der K.K. zoologisch-botanischen Gesellschaft in Wien, 37:511-632.

Quiroz, L. \& J. Valenzuela. 1995. A comparison of ground ant communites in a tropical rainforest and adjacent grassland in Los Tuxtlas, Veracruz, México. Southwestern Entomologist, 20: 203-213.

Recibido: 6 de febrero de 2008

Aceptado: 7 de noviembre de 2008 